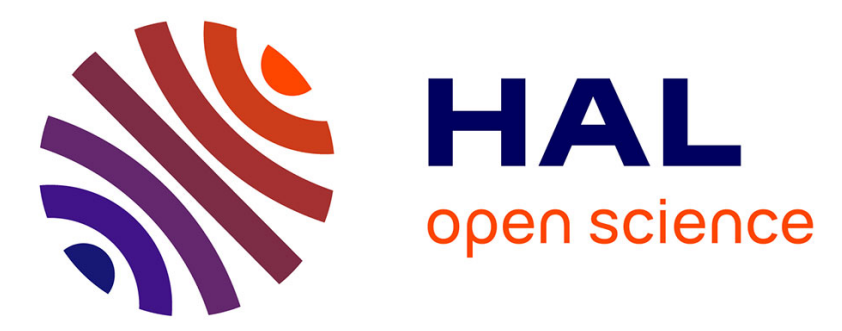

\title{
L'éthique environnementale comme travail du soi relationnel
}

Rémi Beau

\section{To cite this version:}

Rémi Beau. L'éthique environnementale comme travail du soi relationnel: la voie des pratiques de l'ordinaire". Cités: Philosophie, politique, Histoire, 2018, 76 (4), pp.109. 10.3917/cite.076.0109 . hal-01975031

\section{HAL Id: hal-01975031 \\ https://hal.science/hal-01975031}

Submitted on 9 Jan 2019

HAL is a multi-disciplinary open access archive for the deposit and dissemination of scientific research documents, whether they are published or not. The documents may come from teaching and research institutions in France or abroad, or from public or private research centers.
L'archive ouverte pluridisciplinaire HAL, est destinée au dépôt et à la diffusion de documents scientifiques de niveau recherche, publiés ou non, émanant des établissements d'enseignement et de recherche français ou étrangers, des laboratoires publics ou privés. 


\section{"L'éthique environnementale comme travail du soi relationnel: la voie des pratiques de l'ordinaire".}

(Rémi Beau, Université Paris 1 Panthéon-Sorbonne)

\section{Introduction}

"Everything is connected to Everything else ", tel était le slogan de l'écologie des années 1970 popularisée par le biologiste américain Barry Commoner ${ }^{1}$. Rendant visibles des relations de causalité, qui avaient pour l'essentiel été ignorées, la science écologique venait de mettre en pleine lumière les boucles de rétroaction qui ne permettaient plus de penser les actions humaines isolées de leur environnement. De ce point de vue, il s'agissait de souligner que les êtres humains n'agissent jamais seuls, mais toujours par le biais d'interactions avec d'autres êtres qui font système et que les dégradations environnementales, du fait même de cette nature systémique, menaçaient nécessairement en retour les populations humaines.

Mais que faire de ce "fait de l'interdépendance " ? Au-delà de la vision d'une menace généralisée, la compréhension de cette vulnérabilité commune que dévoilait l'interdépendance peut aussi indiquer une voie permettant de redonner du sens à l'action écologique dans le contexte du changement global.

Afin de mettre en lumière cette positivité de l'interdépendance, nous montrerons, en nous appuyant sur l'éthique écocentrique développée dans le sillage d'Aldo Leopold, comment celle-ci invite à modifier en profondeur les manières que nous avons de nous représenter nous-mêmes et à développer une conception relationnelle du monde et du soi. Nous nous interrogerons dès lors, à la suite du philosophe norvégien Arne Naess, sur les implications éthiques d'une telle conception d'un soi que l'on décrira comme

\footnotetext{
${ }^{1}$ COMmOneR Barry, The closing circle: nature, man, and technology, Knopf, 1971, $360 \mathrm{p}$.
} 
" écologique ". Qu'est-ce qu'agir en tant que soi relationnel ? De quelle façon cette transformation de soi permet-elle d'accéder à une nouvelle réflexivité sur nos pratiques ? Après avoir décrit quelquesunes des difficultés que soulèvent ces questions, nous examinerons une proposition élaborée dans la perspective du perfectionnisme moral que Stanley Cavell a ouverte à partir des travaux d'Emerson et Thoreau.

\section{De l'interdépendance au soi relationnel}

Bien avant les années 1970, le forestier américain Aldo Leopold avait décrit les implications éthiques du développement de la science écologique. Dans l'Almanach d'un Comté des Sables, il affirmait que l'interdépendance était la condition première de toute éthique. "Toutes les éthiques élaborées jusqu'ici reposent sur un seul présupposé : que l'individu est membre d'une communauté de parties interdépendantes ${ }^{2}$ ". L'éthique ne peut prendre sens que lorsqu'elle porte sur les actions d'êtres en relation les uns par rapport aux autres et dont le sort individuel est en partie lié à celui de la communauté d'êtres interdépendants à laquelle ils appartiennent. Or, dès lors qu'il apparaît que cette interdépendance n'engage pas simplement les êtres humains, mais aussi la terre, les animaux et les plantes, l'inclusion des êtres non humains dans l'éthique devrait $s^{\prime} i m p o s e r$ comme une " nécessité écologique ${ }^{3}$.

Est-ce à dire que Leopold donne une justification purement prudentielle au développement d'une éthique environnementale ? Ce serait oublier que pour lui, il est « inconcevable qu'une relation éthique à la terre puisse exister sans amour, sans respect, sans admiration pour elle et sans une grande considération pour sa valeur ${ }^{4}$ ». Une éthique environnementale ne saurait donc se réduire à la recherche d'un intérêt bien compris par les membres humains de la communauté biotique. La compréhension de cette interdépendance

\footnotetext{
${ }^{2}$ LEOPOLD Aldo, Almanach d'un comté des sables: suivi de quelques croquis, traduit par Anna GIBSON, Paris, Flammarion, coll. « GF », n 1060, 2000, p. 258.

${ }^{3}$ Ibid., p. 257.

${ }^{4}$ Ibid., p. 282.
} 
généralisée doit éveiller une "conscience écologique ${ }^{5}$ ", donner naissance à un "sentiment de fraternité avec les autres créatures ; un désir de vivre et de laisser vivre ${ }^{6}$ ». Sous cet angle, la land ethic leopoldienne révèlerait bien en quelque sorte la dimension normative du « fait de l'interdépendance ».

Prolongeant Leopold, le philosophe Baird Callicott soutient que prendre la pleine mesure des implications métaphysiques du développement de l'écologie scientifique conduit plus loin encore. Pour ce dernier, cela consiste à remettre en cause l'existence même de l'individu envisagé comme un atome social ${ }^{7}$. De ce point de vue, l'écologie ne dévoilerait pas simplement l'interdépendance des individus, mais plus profondément leur constitution relationnelle. La radicalité théorique de l'écologie tiendrait ainsi dans l'affirmation que nous $n^{\prime}$ avons jamais été les individus que nous croyions être ${ }^{8}$. Introduite dans le champ de la pensée sociale, la thèse écologique de l'interdépendance généralisée conduirait donc, en définitive, à transformer nos manières de nous représenter nous-mêmes et à adopter une conception relationnelle du soi.

Stimulante sur le plan intellectuel, cette hypothèse soulève néanmoins une interrogation quant à la manière dont s'effectue ce passage de la description scientifique des interconnexions entre les êtres à l'expérience qui serait faite " en première personne " de sa nature relationnelle ${ }^{9}$. Callicott met en place un modèle explicatif dans lequel le développement des sciences incarne l'agent central de la transformation individuelle et sociale. Mais, si ce modèle qui

\footnotetext{
${ }^{5}$ LEOPOLD Aldo, La conscience écologique, Wildproject Editions, 2013, 226 p.

${ }^{6}$ LeOPOLD Aldo, Almanach d'un comté des sables, op. cit., p. 145.

${ }^{7}$ CALLICOTT John Baird, "The Metaphysical Implications of Ecology ", Environmental Ethics, 1986, vol. 8, n 4, pp. 301-316.

${ }^{8}$ GILBERT Scott F., SAPP Jan et TAUBER Alfred I., " A symbiotic view of life: we have never been individuals ", The Quarterly review of biology, 2012, vol. 87, n 4, pp. 325-341.

${ }^{9}$ LARRERE Catherine, « Une écologie en première personne pour habiter la Terre. Écologie et littérature ", in La mésologie, un autre paradigme pour I'anthropocène?: autour et en présence d'Augustin Berque, Paris, Hermann, 2018, p.
} 
conduit de la science à l'éthique peut se justifier théoriquement ${ }^{10}$, il lui reste à convaincre sur le plan historique, c'est-à-dire à donner quelques arguments solides nous permettant de croire que la description objective de l'interdépendance généralisée va réellement conduire les individus à transformer la représentation qu'ils ont d'eux-mêmes et à prendre pour nouveaux repères moraux dans leurs actions une éthique environnementale relationnelle. Or, Callicott dit peu de choses sur les ressorts de cette transformation individuelle.

\section{De l'ontologie à l'éthique relationnelles : la voie de I'ordinaire}

Comment passer d'une conception relationnelle du monde à une action menée en tant que sujet relationnel, ou autrement dit d'une ontologie à une éthique relationnelles? Le philosophe norvégien Arne Naess s'est explicitement confronté à la tâche difficile de décrire la façon dont les individus pouvaient se mettre en capacité de vivre l'interconnexion en première personne ${ }^{11}$. Pour Naess, ce processus, qui passe par l'édification par chacun de sa propre " écosophie " - cette précision marquant l'attachement de l'auteur au pluralisme -, apparaît comme une maturation progressive de l'ego vers ce qu'il appelle le "Soi écologique ». Cette proposition a toutefois soulevé des critiques importantes. En ouvrant la sphère égotique afin de donner au soi un horizon relationnel, Naess ne finitil pas par dissoudre les sujets dans un " océan de la continuité ${ }^{12}$ " ? Autrement dit, le prix de cette sortie de l'ego n'est-ce pas une sorte de fusion et d'indifférenciation générale qui pourraient déboucher sur un holisme politique et rendraient, de surcroit, impossible toute éthique écologique ${ }^{13}$ ?

Sur le plan théorique, la conception relationnelle du soi apporte une réponse d'une simplicité désarmante à la question éthique de

\footnotetext{
${ }^{10}$ CALLICOTT John Baird, « Primauté de la philosophie naturelle sur la philosophie morale ", Les Carnets des Cahiers Philosophiques, 28 novembre 2011, pp. 41-62. ${ }^{11}$ NAESS Arne, Ecology, Community, and Lifestyle: Outline of an ecosophy, traduit par David ROTHENBERG, Cambridge University., 1989.

12 Plumwood Val, Feminism and the Mastery of Nature, Taylor \& Francis, 2002, p. 3.

13 PLumwood Val, " La nature, le moi et le genre ", Ecologie \& politique, traduit par Hicham-Stéphane AfEISSA, 26 février 2014, N48, nº 1, pp. 143-175.
} 
l'accord des intérêts égoïstes et altruistes. Comme le résume Baird Callicott, en affirmant que le " monde est notre propre corps", "la vision relationnelle de soi transforme l'égoïsme en écologisme ${ }^{14}$ ». Mais, cette expansion d'un soi qui devient coextensif avec le monde pourrait bien conduire, à l'inverse, au recouvrement de l'ensemble diversifié des intérêts non humains par ceux d'un ego humain surdimensionné et donc paradoxalement à une forme achevée d'anthropocentrisme.

Comment penser cette ouverture relationnelle sans suivre une montée en abstraction qui mène trop précipitamment de l'atome social à la totalité du monde? La solution est indiquée par la philosophe australienne Val Plumwood: il faut se tourner vers les pratiques concrètes par lesquelles se construisent des soi écologiques, c'est-à-dire des pratiques qui favorisent l'intégration consciente par les sujets sociaux de relations nouées avec des êtres non humains et contribuent à la formation de leur identité relationnelle ${ }^{15}$. Ce faisant, elle nous semble indiquer la voie d'une éthique de l'ordinaire qui fait de l'exploration du proche la possibilité d'un renouvellement de la pensée morale.

Investir la pensée américaine de l'ordinaire qui remonte à Emerson et Thoreau et que Stanley Cavell a dépeinte sous la figure du " perfectionnisme moral " permet-il de mieux résister à la " pulsion de généralité " que pourrait éveiller la nécessité écologique de "sortir de soi » ? Celle-ci paraît offrir, en effet, à l'éthique environnementale une voie qui permet, non pas de résoudre la tension entre le soi et le monde, mais d'en faire le catalyseur du perfectionnement moral. A ce titre, le départ à Walden de Thoreau est exemplaire : il n'est en rien la manifestation d'une volonté de repli sur soi ${ }^{16}$, mais le commencement d'une expérience visant à "sortir hors de soi ", l'engagement d'une "conversation avec le

\footnotetext{
${ }^{14}$ CALlicott John Baird, Ethique de la terre, Paris, Éd. Wildproject, coll. « Domaine sauvage ", 2011, vol.1/, p. 103.

${ }^{15}$ Plumwood Val, Feminism and the Mastery of Nature, op. cit., p. 182-189.

${ }^{16}$ ThOREAU Henry David, Walden ou la Vie dans les bois, traduit par Louis FABULET, Paris, Gallimard, coll. "Collection I'Imaginaire », n² 239, 1990, 332 p.
} 
dehors ${ }^{17}$ sans laquelle toute réalisation de soi est impossible. Mais cette nécessité du dehors n'est pas pour Thoreau celle du «Big Outside " ou de la nature spectaculaire des grands espaces, elle se satisfait mieux par l'observation attentive et les transactions avec les êtres qui se situent à proximité de l'étang de Walden.

Ainsi, pas plus qu'il ne se dévoile comme une simple découverte scientifique, le soi écologique ne se révèle dans l'immédiateté d'une identification avec le monde. II est un soi dont la constitution n'est jamais achevée et dont les transformations successives deviennent l'objet même d'une éthique environnementale pensée comme un travail répété de mise au jour, d'actualisation et de création de nouvelles relations avec ses co-habitants humains et non-humains.

\section{Des pratiques de transformation du soi écologique}

Approcher l'éthique environnementale sous l'angle du perfectionnisme moral indique une voie de transition pluraliste dans laquelle les individus peuvent engager un travail de transformation qui vise à la réalisation d'un soi toujours plus écologique, c'est-à-dire toujours mieux relié au monde et à ses habitants. Cette approche invite à investir le terrain de la question environnementale munis d'une conception active et pratique de l'éthique où les transformations de soi et de la société s'appellent l'une, l'autre ${ }^{18}$. Elle entend ainsi mettre à la disposition des individus des techniques ou des exercices concrets actualisant ce que Pierre Hadot appelait un « mode de vie philosophique ${ }^{19}$.

Dans un ouvrage proposant une lecture croisée de Foucault, Hadot et Cavell, Daniele Lorenzini propose d'appeler «techniques de l'ordinaire » cette "série d'exercices, de techniques ou de pratiques

\footnotetext{
${ }^{17}$ ZASK Joëlle, "Sortir hors de soi ", in La voix et la vertu, Presses Universitaires de France, 2010, pp. 397-416.

${ }^{18}$ CAVELL Stanley, " Conditions nobles et ignobles. La constitution du perfectionnisme émersonien [1990] ", in Qu'est-ce que la philosophie américaine ?, Paris, Gallimard, 2009, p. 209.

${ }^{19}$ HADOT Pierre, Exercices spirituels et philosophie antique, Albin Michel, 2014, $342 \mathrm{p}$.
} 
que les individus [utilisent] afin de donner une forme à leur existence quotidienne et de transformer leur rapport à eux-mêmes, aux autres et au monde 20 ». Parmi ces dernières, nous voudrions extraire deux techniques qui nous semblent à même de contribuer à la réalisation d'un soi écologique. La première appartient à l'ensemble des "techniques de l'attention" dont l'objectif est de produire une conversion du regard afin de rendre visible ce qui échappe ordinairement à notre vue. II s'agit plus précisément de développer ce que Sandra Laugier a décrit comme une "attention au particulier ${ }^{21}$, une manière de percevoir dans l'environnement proche de nos vies quotidiennes l'importance de relations inaperçues ou négligées jusqu'alors ${ }^{22}$. C'est une manière active de réexaminer ce que Thoreau appelait les "faits essentiels de la vie » : se nourrir, se vêtir, se chauffer, se loger, converser avec ses voisins humains ou non. Cette voie de l'ordinaire est celle d'une enquête menée en première personne consistant à se demander de quelles façons nos actions quotidiennes nous relient aux êtres non humains ${ }^{23}$.

Du point de vue de la réflexion écologique, cela conduit à rediriger un regard qui a longtemps été fixé sur les espaces naturels les plus éloignés des hommes vers le sol proche de l'ordinaire ${ }^{24}$, y compris celui des espaces urbains. Prêter attention aux êtres non humains qui habitent ces espaces, c'est s'intéresser à des animaux souvent déconsidérés ${ }^{25}$, mais aussi aux herbes de la ville qu'Aldo Leopold, luimême, ne dépréciait en aucun cas ${ }^{26}$, ou encore au monde des

\footnotetext{
${ }^{20}$ LORENZINI Daniele, Éthique et politique de soi. Foucault, Hadot, Cavell et les techniques de l'ordinaire, Vrin., Paris, 2015, p. 11.

${ }^{21}$ LAUGIER Sandra, "Care et perception, l'éthique comme attention au particulier ", in Le souci des autres, éthique et politique du care, Paris, Ed. de l'EHESS, 2006, pp. 317-348.

${ }^{22}$ Voir également Citton Yves, Pour une écologie de l'attention, Le Seuil, 2014, 303 p..

${ }^{23}$ Sur cette idée de cultiver son attention écologique, voir également PELLUCHON Corine, Ethique de la considération, Paris, Seuil, 2018, p. 214-217..

${ }^{24}$ BEAU Rémi, « From Wilderness to Ordinary Nature », Environmental Ethics, 2015, vol. $37, n^{\circ} 4$, pp. $425-443$.

${ }^{25}$ Nagy Kelsi et Johnson (II) Phillip David, Trash Animals: How We Live with Nature's Filthy, Feral, Invasive, and Unwanted Species, University of Minnesota Press, 2013, $314 \mathrm{p}$.

${ }^{26}$ LEOPOLD Aldo, Almanach d'un comté des sables, op. cit., p. 222.
} 
insectes 27. Mais, s'interroger sur la qualité de ses relations constitutives, ce peut être également se demander si le morceau de viande que je mange me lie de façon satisfaisante à l'animal dont il est issu, à l'environnement dans lequel il a été élevé et à son éleveur ou encore si le légume, la céréale ou le fruit que j'ingère me relient bien à la terre cultivée et à son cultivateur. On tend parfois à minorer la capacité de déstabilisation de ce type d'approche en la réduisant à une "écologie des petits gestes " ${ }^{28}$. C'est manquer la dimension critique d'un tel travail sur soi et l'articulation entre l'éthique et la politique qu'il permet d'établir, car les transformations successives de soi passent, comme chez Thoreau, par le développement de contre-conduites ${ }^{29}$ ou de conduites de résistance qui œuvrent bien à la transformation de la société.

En outre, ce perfectionnisme environnemental suggère une manière convaincante d'articuler l'enquête subjective et l'appréhension scientifique de l'interdépendance. C'est ce que permet de comprendre la convocation d'un second exercice mentionné par Daniele Lorenzini et qui s'inscrit dans les «techniques de la pensée ${ }^{30}$. Celui-ci est défini comme un " exercice du regard», qui ne consiste pas à observer horizontalement son environnement proche, mais à adopter un " regard d'en haut " ou " en troisième personne ${ }^{31}$. II s'agit de travailler à l'amélioration de sa connaissance de la nature et du monde afin de mieux se situer dans ce vaste tissu de relations. Hadot pensait de ce point de vue à l'étude de la physique ${ }^{32}$, mais cet exercice ne nous semble pas moins pertinent en ce qui concerne l'étude de l'écologie scientifique. S'il s'oppose ainsi

\footnotetext{
${ }^{27}$ RAFFlES Hugh, Insectopedie, traduit par Matthieu DuMONT, Marseille, Wildproject Editions, 2016, $440 \mathrm{p}$.

${ }^{28}$ Aсот Pascal, L'écologie de la libération: les dégradations de l'environnement sont des crimes contre l'humanité, Le Temps des cerises, 2017, 138 p.

${ }^{29}$ LORENZINI Daniele, Éthique et politique de soi. Foucault, Hadot, Cavell et les techniques de l'ordinaire, op. cit., p. 13.

${ }^{30}$ Ibid., p. 125-146.

${ }^{31}$ LARRERE Catherine, "Une écologie en première personne pour habiter la Terre. Écologie et littérature ", op. cit.

${ }^{32}$ HADOT Pierre, Exercices spirituels et philosophie antique, op. cit., p. 359-360.
} 
au scientisme ${ }^{33}$, le perfectionnisme environnemental n'en aspire pas moins à être une éthique écologiquement informée.

Tout en reconnaissant qu'il serait absurde de demander à tous ses concitoyens d'obtenir un doctorat en écologie, Leopold soulignait déjà, néanmoins, que leur absence de culture écologique était un obstacle sérieux au développement d'une éthique environnementale. Sous cet angle, l'approche perfectionniste propose un moyen concret d'œuvrer à la transformation de soi. Loin d'opposer l'attention au particulier et le regard objectif sur les relations entre les êtres, elle soutient ainsi qu'améliorer l'acuité de ces deux types de regard contribue à la réalisation d'un soi écologique.

\section{Conclusion}

Les éthiques environnementales ont aujourd'hui près de cinquante années d'existence. Loin d'être négligeables sur le plan pratique, puisqu'elles ont contribué à introduire dans le débat public certaines des questions que pose notre façon de nous conduire avec les êtres non-humains, elles continuent néanmoins à chercher le moyen d'œuvrer plus efficacement à la transition écologique des sociétés contemporaines. Dans cette optique, cette variété du perfectionnisme moral nous semble constituer l'une des voies permettant de prolonger le développement de l'éthique environnementale, mais aussi de faire fructifier un certain nombre des réflexions menées par les auteurs classiques de la discipline. C'est ainsi qu'il nous semble qu'interpréter la land ethic leopoldienne, en particulier les descriptions des pratiques que le forestier conduit dans les espaces naturels proches de sa ferme du Wisconsin, comme une forme de perfectionnisme environnemental ${ }^{34}$ restitue à la pensée de l'écologiste américain toute sa force de transformation individuelle et collective. Arrimée à une pensée de l'ordinaire, celle-ci redonne un

\footnotetext{
${ }^{33}$ Scientisme qui affleure souvent chez Callicott, voir sa volonté de réhabiliter une science de l'éthique dans CALLICOTT John Baird, Thinking Like a Planet: The Land Ethic and the Earth Ethic, Oxford University Press, 2014, 402 p.

${ }^{34}$ BEAU Rémi, Ethique de la nature ordinaire: Recherches philosophiques dans les champs, les friches et les jardins, Paris, Publications de la Sorbonne, 2017, 342 p.
} 
horizon proche à l'action écologique, celui de l'environnement quotidien de sujets qui expérimentent de diverses manières la conduite de leur vie en tant que soi écologique. 